\title{
Explicit Non-Adaptive Combinatorial Group Testing Schemes
}

\author{
Ely Porat* Amir Rothschild * $†$
}

\begin{abstract}
Group testing is a long studied problem in combinatorics: A small set of $r$ ill people should be identified out of the whole ( $n$ people) by using only queries (tests) of the form "Does set X contain an ill human?". In this paper we provide an explicit construction of a testing scheme which is better (smaller) than any known explicit construction. This scheme has $\Theta\left(\min \left[r^{2} \log n, n\right]\right)$ tests which is as many as the best non-explicit schemes have. In our construction we use a fact that may have a value by its own right: Linear error-correction codes with parameters $[m, k, \delta m]_{q}$ meeting the Gilbert-Varshamov bound may be constructed quite efficiently, in $\Theta\left(q^{k} m\right)$ time.
\end{abstract}

*Bar-Ilan University, Dept. of Computer Science, 52900 Ramat-Gan, Israel, porately@cs.biu.ac. i

${ }^{\dagger}$ Tel-Aviv University, Dept. of computer science, Tel-Aviv, Israel, rotshch@post.tau.ac.i 


\section{Introduction}

Group testing is an important and well known tool in combinatorics. Due to its basic nature, it has been found to be applied in a vast variety of situations. In 2006 DIMACS has dedicated a special workshop solely for the problem of group testing [16]. A representative instance of group testing considers a set of items, each of which can be either defective or non-defective, and the task is to identify the defective items using the minimum number of tests. Each test works on a group of items simultaneously and returns whether or not that group contains at least one defective item. A group testing algorithm is said to be nonadaptive if all the tests to be performed are specified in advance. A formal definition is given in Section 2.

Group testing has a long history dating back to at least 1943 [26]. In this early work the problem of detecting syphilitic men for induction into the United States military using the minimum number of laboratory tests was considered. While this idea is still relevant today for testing viruses such as HIV, it is only one of the many applications found for group testing: In the effort of mapping genomes, for example, we have a huge library of DNA sequences, and test whether each of them contains a probe from a given set of DNA pieces [6, 15, 45]. Somewhat less conventional uses for group testing were introduced lately in pattern matching algorithms [22, 4] and in streaming algorithms [24]: For instance, [22] solves the problem of searching for a pattern in a text with a bounded number of mismatches. A recent paper about pattern matching in a streaming model even utilize group testing twice in the same algorithm [41]. Additional applications of group testing include: quality control in product testing [44], searching files in storage systems [34], sequential screening of experimental variables [37], efficient contention resolution algorithms for multipleaccess communication [34, 48], data compression [33], software testing [9, 23], DNA sequencing [40] and other applications in computational molecular biology [27, 28, 39, 5].

Consider the situation where there are $n$ items out of which at most $r$ are defective. It has been shown that in this situation any nonadaptive combinatorial group testing (GT) procedure must use $\Omega\left(\min \left[r^{2} \log _{r} n, n\right]\right)$ tests [17]. The best known schemes use $\Theta\left(\min \left[r^{2} \log n, n\right]\right)$ tests [34], and the best known explicit (polynomial time constructable) schemes need as much as $\Theta\left(\min \left[r^{2} \log ^{2} n, n\right]\right)$ tests [34]. In this paper, we present an explicit GT scheme which contains merely $t=\Theta\left(\min \left[r^{2} \log n, n\right]\right)$ tests (the same as the best known non-explicit schemes), and takes $\Theta(r n \log n)$ time to build, which is linear in its representation $\left(\mathcal{O}\left(t \frac{n}{r}\right)\right)$. Hence, this paper closes the gap between the explicit and non-explicit group testing schemes.

\subsection{Error Correction Codes}

An error-correcting code (ECC) is a method for encoding data in a redundant way, such that any errors which are introduced can be detected and corrected (within certain limitations). Suppose Alice wants to send Bob a string of $k$ letters from alphabet of size $q$ using some noisy channel. An $(m, k, d)_{q}$ error-correction code enables Alice to encode her string to an $m>k$ letters string, such that Bob will be able to detect whether the received message has up to $d$ errors, and even decode the message if it has less than $\frac{d}{2}$ errors. A linear code (LC) is an important type of errorcorrection code which allows more efficient encoding and decoding algorithms than other codes. Error-correction codes are used in a vast variety of fields including information transmission, data preservation, data-structures, algorithms, complexity theory, and more.

One of the most important goals of coding theory is finding codes that can detect many errors, 
while having little redundancy. The Gilbert-Varshamov (GV) bound shows this can be done to some extent: We define the rate of a code, $R=\frac{k}{m}$ and the relative distance of a code, $\delta=\frac{d}{m}$. The GV bound asserts that there are codes with $R \geq 1-H_{q}(\delta)-o(1)$ where $H_{q}(p)$ is the $q$-ary entropy function $H_{q}(p)=p \log _{q} \frac{q-1}{p}+(1-p) \log _{q} \frac{1}{1-p}$, and $o(1) \rightarrow 0$ as $m \rightarrow \infty[30,47]$. Though the GV bound is half a century old, no explicit construction of codes meeting it has yet been found. The best known construction takes polynomial time in $q^{m-k}[14]$.

We present a more efficient deterministic construction for linear codes meeting the GV bound. Our construction takes $\Theta\left(q^{k} m\right)$ time. The importance of this result is apparent when constructing codes with low rates; First, for small rates the GV bound is the best known lower bound on the rate and relative distance of a code. Second, the lower the rate, the slower the previously known best construction, and the faster our construction.

\subsection{Previous Results}

Since the problem of group testing was first introduced in 1943, many problems related to it and generalizations of it were considered including: fully-adaptive group testing, two staged group testing and selectors $[19,35,10,21,13,11]$, group testing with inhibitors [29, 25, 12, 10], group testing in a random case where a distribution is given on the searched set $[35,7,8,1,10]$, group testing in the presence of errors [36] and more. Regarding the original problem of group testing, Kautz and Singleton [34] proved the existence of GT schemes of size $\Theta\left(r^{2} \log n\right)$, and showed how to explicitly construct schemes of size $\Theta\left(r^{2} \log ^{2} n\right)$. They also managed to give an explicit construction of schemes of size $\Theta(\log n)$ for the special case $r=2$. Since their work, no asymptotic improvements to the size of the GT scheme were found. One paper succeeded, however, in improving the size of the explicit schemes (but only for constant values of $r$ ): [3] showed how to construct an explicit construction of schemes of size $\Theta\left(r^{2} \log n\right)$ in time polynomial in $n^{r}$.

Regarding error-correction codes the picture is more complexed. The GV bound was first presented by Gilbert in 1952 [30]. He provided a $\Theta\left(q^{m}\right)$ time greedy construction for codes meeting his bound. A few years later Varshamov [47] showed linear codes share this bound and Wozencraft [49] offered a $\Theta\left(q^{m}\right)$ time deterministic construction of such codes. In 1977 Goppa [31] initiated the fruitful study of algebraic geometric codes. Codes eventually found by this study surpass the GV bound for various alphabet sizes and rates [46]. Recently, an explicit, $\Theta\left(m^{3}\right.$ polylog $\left.m\right)$ time construction was given for algebraic geometric codes [42, 43]. The best deterministic construction for alphabet sizes and rates where the GV bound is superior to the algebraic geometric bound, was provided in 1993 by Brualdi and Pless [14]. They presented a poly $\left(q^{m-k}\right)$ construction of binary linear codes meeting the GV bound. Their construction can be easily generalized to deal with larger alphabets. Even under hardness assumptions, no explicit construction of codes meeting the GV bound has yet been found, though an effort presenting some worthy results is given in [18].

\subsection{The Paper Outline}

We start this paper with formal definitions in Section 2, and continue by showing a connection between error-correction codes and group testing schemes in Section 3. Then we immediately move to the main result of the paper in Section 4, showing how to efficiently construct small group testing schemes. This construction for group testing schemes uses our construction of a linear code which is given in Section 5 . 


\section{Problems Definitions}

Definition 2.1 Consider a universe $U$. A family of tests (subsets) $\mathcal{F} \subset \mathcal{P}(U)$ is a group testing scheme of strength $r$ if for any subset $A \subset U$ of size at most $r$, and for any element $x \notin A$, there exist a test $B \in \mathcal{F}$ that distinguishes $x$ from $A$, meaning $x \in B$ while $A \cap B=\varnothing$.

In order to ease reading, we present short notations of an error-correction code and a linear code.

Definition 2.2 An ECC, $\mathcal{C}$, is said to have parameters $(m, k, d)_{q}$ if it consists of $q^{k}$ words of length $m$ over alphabet $\Sigma$ of $q$ elements, and has hamming distance $d$. Such an ECC is denoted as $(m, k, d)_{q}-E C C$.

Definition 2.3 An $[m, k, d]_{q}-L C$ is a special case of an $(m, k, d)_{q}-E C C$ which is over alphabet $\Sigma=\mathbb{F}_{q}$ when the codewords form a linear subspace over $\mathbb{F}_{q}^{m}$. Such a linear code is said to have parameters $[m, k, d]_{q}$. A linear code has a generation matrix $\mathcal{G} \in \mathcal{M}_{m \times k}$ which generates it, meaning $\mathcal{C}=\left\{\mathcal{G} y \mid t \in \mathbb{F}_{q}^{k}\right\}$.

\section{Background}

Our results concerning GT are more natural and straightforward once the combinatorial concepts selection by intersection and strongly-selective family (SSF) [20] have been explained. Selection by intersection means distinguishing an element from a set of elements by intersecting it with another set. More precisely,

Definition 3.1 Given a subset $A \subset U$ of a universe $U$, element $x \in A$ is selected by subset $B \subset U$ if $A \cap B=\{x\}$. An element is selected by a family of subsets $\mathcal{F} \subset \mathcal{P}(U)$ if one of the subsets in $\mathcal{F}$ selects it.

An SSF is a family of subsets that select any element out of a small enough subset of the universe. More precisely,

Definition 3.2 A family $\mathcal{F} \subset \mathcal{P}(U)$ is said to be $(n, r)$-strongly-selective (or $(n, r)$-StS for short) if, for every subset $A \subset U$ of size $|A|=r$, all elements of $A$ are selected by $\mathcal{F}$. We call such a family an $(n, r)-S S F$.

SSFs and GT schemes are strongly connected: On the one hand, an $(n, r+1)$-SSF is a GT scheme of strength $r$, and on the other hand, a GT scheme of strength $r$ in a universe of size $n$ is an $(n, r)$-SSF. For a detailed proof see [34].

In what follows we will focus on SSF constructions. It is important to note that explicit constructions for SSFs give explicit constructions for GT schemes with the same asymptotic behavior. Next we show how to construct an SSF from an ECC, and how good this construction is in different situations. The foundations of some of the ideas we present were developed in an earlier work by Kautz and Singleton on superimposed codes [34]. The context and formalisms that were employed are quite distinct from those we require, the ideas are quite simple and though, we are not aware of this aspect of their work being developed subsequently. Thus, in the following Subsection we will provide full proof for the lemma we claim. 


\subsection{Reducing ECCs to SSFs}

As it turns out, one can build small strongly-selective families from good error-correction codes having large distance. Both the construction and the proof are given in this Subsection. In a few words, the idea behind the construction is that taking a small set of codewords from the ECC and another codeword $w$, there must be positions in which $w$ differs from all the words in this set. This is because $w$ differs from any other word in the code in many positions, and so, in a small set of codewords, there must be some shared positions in which all codewords differ from $w$. Therefore we'll get an SSF if we first translate elements of $[n]$ to codewords, and second, find tests which isolate a codeword $w$ from a set of codewords if it differs from this set in a certain position. We construct such tests by assembling a test for each possible letter in each possible position in the text. A detailed construction follows.

Suppose $\mathcal{C}=\left\{w_{1}, \ldots, w_{n}\right\}$ is an $\left(m, \log _{q} n, \delta m\right)_{q}$-ECC. The constructed SSF, $\mathcal{F}(\mathcal{C})$, will be assembled from all the sets of indexes of codewords that have a certain letter in a certain position. More accurately, for any $p \in[m]$ and $v \in[q]$, define $s_{p, v}=\left\{i \in[n] \mid w_{i}[p]=v\right\}$. Define $\mathcal{F}(\mathcal{C})$ as the set of all such $s_{p, v}$-s: $\mathcal{F}(\mathcal{C})=\left\{s_{p, v} \mid p \in[m]\right.$ and $\left.v \in[q]\right\}$.

The size of $\mathcal{F}(\mathcal{C})$ is at most $m q$. Notice that this construction may be performed in time $\Theta(n m)$ (linear in the size of the representation of $\mathcal{F}$ ) using Algorithm 1.

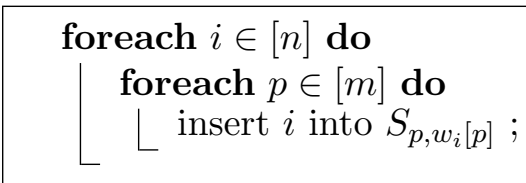

Algorithm 1: Constructing an SSF from an ECC

The following Lemma shows that this construction really does result in a small SSF, and more specifically, that $\mathcal{F}(\mathcal{C})$ is $\left(n,\left\lceil\frac{1}{1-\delta}\right\rceil\right)-\mathrm{StS}$.

Lemma 3.1 Let $\mathcal{C}$ be an $\left(m, \log _{q} n, \delta m\right)-E C C$. Then the family $\mathcal{F}(\mathcal{C})$ is $\left(n,\left\lceil\frac{1}{1-\delta}\right\rceil\right)-S t S$.

Proof: Let $r=\left\lceil\frac{1}{1-\delta}\right\rceil$. Let $i_{1}, \ldots, i_{r} \in[n]$ be any $r$ distinct indexes in [n]. W.L.O.G. we prove that $i_{1}$ is selected from $\left\{i_{1}, \ldots, i_{r}\right\}$ by $\mathcal{F}(\mathcal{C})$. For any $j \neq 1$, the number of positions $p \in[m]$ where $w_{i_{j}}[p]=w_{i_{1}}[p]$ is at most $(1-\delta) m$. Thus, the number of positions where $w_{i_{1}}[p] \in\left\{w_{i_{2}}[p], \ldots, w_{i_{r}}[p]\right\}$ is at most $(r-1)(1-\delta) m<m$. Therefore, there exist a position $p$ where $w_{i_{1}}[p] \notin\left\{w_{i_{2}}[p], \ldots, w_{i_{r}}[p]\right\}$. This means that $i_{1} \in s_{p, w_{i_{1}}[p]}$ while all other $i_{j}$-s are not. Thus, $i_{1}$ is selected by $s_{p, w_{i_{1}}[p]}$.

\section{Main Theorem}

Theorem 1 Let $n$ and $r$ be positive integers. It is possible to construct a $(n, r)$-SSF of size $\Theta\left(\min \left[r^{2} \log n, n\right]\right)$ in $\Theta(r n \log n)$ time.

Proof: If $r^{2} \log n \geq n$, simply return the $n$ tests $\{i\}_{i=1}^{n}$. We continue the proof assuming that $r^{2} \log n<n$. Set $\delta=\frac{r-1}{r}$ (which is equivalent to $r=\frac{1}{1-\delta}$ ), $q=2 r, k=\log n$ and $m=\frac{k}{1-H_{q}(\delta)}=$ $\Theta(r k)$. 
Use Theorem 2 to construct an $[m, k, \delta m]_{q}-\mathrm{LC}$ in time $\Theta(n m)$. This is possible since $k \leq$ $\left(1-H_{q}(\delta)\right) m$.

According to Lemma 3.1, we can now construct an $(n, r)$-SSF of size $m q=\Theta\left(r^{2} \log n\right)$. The time this construction will take is $\Theta(n m)=\Theta(r n \log n)$.

\section{$5 \quad$ Meeting the Gilbert-Varshamov bound more Efficiently}

In this Section we demonstrate a deterministic construction of LCs which meet the GV bound. We developed this deterministic algorithm by taking a random algorithm and derandomizing it using the method of conditional probabilities (a full discussion concerning this method is given in [2]). Using this method requires the random algorithm to have several non-trivial attributes. First, there need to be a goal function goal : LinearCodes $\rightarrow \mathbb{R}$ which returns a large result whenever the random algorithm fails. Second, this function has to have low expectation - lower than the minimum value returned by it when the algorithm fails. Third, the random selections of the algorithm have to be divided into stages with a small number of options to choose from in each. Finally, there should be an efficient algorithm for calculating in each stage of the algorithm the option minimizing the expectation of goal given all the selections done until that point. In Subsection 5.1 we'll show the random algorithm, present the goal function goal, show that the algorithm fails iff goal $(\mathcal{G}) \geq 1$ (where $\mathcal{G}$ is the generation matrix returned by the algorithm), and show that $E($ goal $)<1$. In Subsection 5.2 we'll present the derandomized algorithm more accurately, showing how to divide it to the small stages. We'll also prove it should work, and show how to calculate the option minimizing the expectation of goal in each stage. We'll finish this Subsection having an algorithm taking time polynomially in the complexity we desire, we improve it in Subsection 5.3 to acquire the desired complexity.

\subsection{The Probabilistic Algorithm}

Algorithm 2 is a standard probabilistic algorithm for building linear codes with rate meeting the GV bound.

Input: $m, k \in \mathbb{N}, \delta \in[0,1]$ s.t. $k \leq\left(1-H_{q}(\delta)\right) m$

Pick entries of the $m \times k$ generator matrix $\mathcal{G}$ uniformly and independently at random from $\mathbb{F}_{q}^{m \times k}$

Output: $\mathcal{G}$

Algorithm 2: Probabilistic Construction of a Linear Code

Definition 5.1 Given a codeword $x$ of length $m$, and a distance parameter $\delta \in[0,1]$, we define $\mathcal{B}_{\delta}(x)$ as the bad event that the weight of $x$ is less than $\delta m, \omega(x)<\delta m$. By abuse of notation we refer to $\mathcal{B}_{\delta}(x)$ also as the indicator of the same event.

If we manage to choose a code with no bad event (not considering the 0 codeword, of course), than the weight of the generated code is larger then $\delta m$ and the algorithm succeeds. Therefore, our goal function will be $\operatorname{goal}(\mathcal{G})=\sum_{0 \neq y \in \mathbb{F}_{q}^{k}} \mathcal{B}_{\delta}(\mathcal{G} y)$. The algorithm succeeds iff $\operatorname{goal}(\mathcal{G})=0$. We now need to show that $E$ (goal) is small. Therefore, we are interested in proving that the probability of a bad event is sufficiently small: 
Lemma 5.1 Let $y$ be a nonzero vector in $\mathbb{F}_{q}^{k}$. Let $\mathcal{G}$ be a random generator matrix chosen according to algorithm 2. Then $\log _{q}\left(\operatorname{Pr}\left(\mathcal{B}_{\delta}(\mathcal{G} y)\right)\right) \leq-m\left(1-H_{q}(\delta)\right)$.

Proof: It is easy to see that $x=\mathcal{G} y$ is a random vector in $\mathbb{F}_{q}^{m}$. Therefore, $\omega(x)$ is binomially distributed; $\omega(x) \sim B\left(m, 1-\frac{1}{q}\right)$. Chernoff bound [32] states that

$$
\operatorname{Pr}\left(\mathcal{B}_{\delta}(x)\right)=\operatorname{Pr}(\omega(x) \leq \delta m) \leq\left(\left(\frac{\frac{1}{q}}{1-\delta}\right)^{1-\delta}\left(\frac{1-\frac{1}{q}}{\delta}\right)^{\delta}\right)^{m}
$$

Extracting logarithm from the former expression and simplifying it we attain

$$
\begin{aligned}
\log _{q}\left(\operatorname{Pr}\left(\mathcal{B}_{\delta}(x)\right)\right) & \leq m\left((1-\delta)\left(-1-\log _{q}(1-\delta)\right)+\delta\left(\log _{q}\left(1-\frac{1}{q}\right)-\log _{q} \delta\right)\right) \\
& =-m\left(1-H_{q}(\delta)\right)
\end{aligned}
$$

We will now show that for an appropriate choice of parameters, the expected number of bad events, $E($ goal $)$, is smaller then 1 .

Lemma 5.2 Suppose $\mathcal{G}$ is a random generator matrix chosen according to algorithm 2. Suppose that $k \leq\left(1-H_{q}(\delta)\right) m$. Then $E$ (goal) $<1$.

Proof: By linearity of the expectation

$$
E(\text { goal })=E\left(\sum_{y \neq 0} \mathcal{B}_{\delta}(\mathcal{G} y)\right)=\sum_{y \neq 0} E\left(\mathcal{B}_{\delta}(\mathcal{G} y)\right)=\sum_{y \neq 0} \operatorname{Pr}\left(\mathcal{B}_{\delta}(\mathcal{G} y)\right)
$$

Next, employ Lemma 5.1 to acquire that

$$
E(\text { goal }) \leq\left(q^{k}-1\right) q^{-m\left(1-H_{q}(\delta)\right)}<q^{k-m\left(1-H_{q}(\delta)\right)}
$$

And finally, use our assumption $k \leq\left(1-H_{q}(\delta)\right) m$ to achieve the desired result

$$
E(\text { goal })<1
$$

\subsection{Derandomizing the Algorithm}

Next we will show how to derandomize the algorithm. Algorithm 3 will determine the entries of the generation matrix one by one, while trying to minimize the expectation of the number of bad events, goal. 
Input: $m, k \in \mathbb{N}, \delta \in[0,1]$ s.t. $k \leq\left(1-H_{q}(\delta)\right) m$

Initialize $\mathcal{G}$ to be an $m \times k$ matrix;

foreach $i \in[m]$ do

foreach $j \in[k]$ do

Set $\mathcal{G}[i, j]$ so as to minimize the expected value of $\operatorname{goal}(\mathcal{G})$ given all the values of $\mathcal{G}$ chosen so far;

Output: $\mathcal{G}$

Algorithm 3: Finding a code having no Bad Events

Two questions arise from the above description of the algorithm: First, will this algorithm find a code with no bad events? Second, how can we find the value of $\mathcal{G}[i, j]$ in each step of the algorithm?

The answer to the first question is, of course, positive. The presented algorithm works according to the derandomization scheme of conditional probabilities, and so, the number of bad events in the returned solution will be no more than the expectation of this number before fixing any of the letters. We'll delve into the proof after introducing some additional notations concerning the algorithm:

Definition 5.2 We assert that the algorithm is in step- $(i, j)$ when it is about to choose the entry $(i, j)$ in $\mathcal{G}$. We denote the step following $(i, j)$ by $(i, j)+1$.

Definition 5.3 $S T_{(i, j)}$ will denote the state of the matrix $\mathcal{G}$ at step $(i, j)$ - i.e. which entries have been fixed to which values.

Lemma 5.3 The above algorithm will find a code no bad events, i.e. $\operatorname{goal}(\mathcal{G})=0$.

Proof: Suppose the algorithm is in some step $(i, j)$.

$$
\operatorname{Pr}\left(\mathcal{B}_{\delta}(\mathcal{G} y) \mid S T_{(i, j)}\right)=\frac{1}{q} \sum_{v \in \Sigma} \operatorname{Pr}\left(\mathcal{B}_{\delta}(\mathcal{G} y) \mid S T_{(i, j)}, \mathcal{G}[i, j]=v\right)
$$

Consequently,

$$
\begin{aligned}
E\left(\text { goal } \mid S T_{(i, j)}\right) & =E\left(\sum_{y \neq 0} \mathcal{B}_{\delta}(\mathcal{G} y) \mid S T_{(i, j)}\right) \\
& =\sum_{y \neq 0} \operatorname{Pr}\left(\mathcal{B}_{\delta}(\mathcal{G} y) \mid S T_{(i, j)}\right) \\
& =\frac{1}{q} \sum_{v \in \Sigma} \sum_{y \neq 0} \operatorname{Pr}\left(\mathcal{B}_{\delta}(\mathcal{G} y) \mid S T_{(i, j)}, \mathcal{G}[i, j]=v\right) \\
& \geq \min _{v \in \Sigma} \sum_{y \neq 0} \operatorname{Pr}\left(\mathcal{B}_{\delta}(\mathcal{G} y) \mid S T_{(i, j)}, \mathcal{G}[i, j]=v\right) \\
& =\sum_{y \neq 0} \operatorname{Pr}\left(\mathcal{B}_{\delta}(\mathcal{G} y) \mid S T_{(i, j)+1}\right) \\
& =E\left(\sum_{y \neq 0} \mathcal{B}_{\delta}(\mathcal{G} y) \mid S T_{(i, j)+1}\right) \\
& =E\left(\text { goal } \mid S T_{(i, j)+1}\right)
\end{aligned}
$$


Therefore, if the values of the entries are chosen one by one, so as to minimize the expectation of goal, this value can not increase. Since this value is smaller then 1 in the beginning according to Lemma 5.2, it follows that it is smaller then 1 in the end. But at the end all entries are chosen, and hence the value of goal will be exactly the number of bad events that hold for the codewords we have chosen. This number must be an integer, hence, it is 0 .

The answer to the second question, regarding how to find what the value of $\mathcal{G}[i, j]$ should be, requires additional work. It would be convenient to order the vectors $y \in \mathbb{F}_{q}^{k}$ according to the lexicographic order, setting $y_{\ell}$ to be the $\ell$-s vector according to the lexicographic order.

We need to know for any codeword the number of positions in which it vanishes, at each step of the algorithm. For this purpose maintain an array $A$ of $q^{k}$ entries throughout the algorithm. Entry $A[\ell]$ in this array will hold the number of positions in which the code-word $\mathcal{G} y_{\ell}$ vanished so far. Maintaining this array will require overall $\Theta\left(m q^{k}\right)$ time. This is due to the fact that in each step $(i, j)$ we only need to consider changing the values $A\left[y_{\ell}\right]$ for $q^{j-1} \leq \ell<q^{j}$ since the only letters we fixed during this step belong to these words. We claim that the number of position where the word $\mathcal{G} y_{\ell}$ vanishes determines the conditioned probability of $\mathcal{B}_{\delta}\left(y_{\ell}\right)$.

Lemma 5.4 Consider a codeword $\mathcal{G} y_{\ell}$ for which all entries up to $i$ were fixed (by the entries selected in $\mathcal{G})$, and entries $i$ to $m$ were not fixed yet $\left(\forall t \leq i:\left(\mathcal{G} y_{\ell}\right)[t]=\right.$ fixed $\left.[t]\right)$. Also suppose that until now, $\mathcal{G} y_{\ell}$ doesn't vanish on exactly $c$ positions $(c=\mid\{t \leq i \mid$ fixed $[t] \neq 0\} \mid)$. Then $\omega\left(y_{\ell}\right)-c \sim$ $B\left(m-i, 1-\frac{1}{q}\right)$, and $\operatorname{Pr}\left(\mathcal{B}_{\delta}\left(\mathcal{G} y_{\ell}\right) \mid \forall t \leq i:\left(\mathcal{G} y_{\ell}\right)[t]=\right.$ fixed $\left.[t]\right)$ is the probability that such a binomial variable will be smaller than $\delta m-c$.

Proof: Any entry which wasn't fixed in $\mathcal{G} y_{\ell}$, has a probability of $1-\frac{1}{q}$ to vanish. The entries in $\mathcal{G} y_{\ell}$ are independent of one another, and thus, $\omega\left(\mathcal{G} y_{\ell}\right)-c \sim B\left(m-i, 1-\frac{1}{q}\right)$.

Now, in step $(i, j)$, For any codeword $\mathcal{G} y_{\ell}$ s.t. $q^{j-1} \leq \ell<q^{j}$, we can calculate the probabilities $\operatorname{Pr}\left(\mathcal{B}_{\delta}\left(y_{\ell}\right) \mid S T_{(i, j)}, \mathcal{G}[i, j]=v\right)$ for all $v \in[q]$ in $\operatorname{poly}\left(q^{k}, m\right)$ time using Lemma 5.4. Consequently, we can calculate all the expectations $E\left(\sum_{q^{i-1} \leq \ell<q^{i}} \mathcal{B}_{\delta}\left(y_{\ell}\right) \mid S T_{(i, j)}, G[i, j]=v\right)$ for all $v \in[q]$ in $\operatorname{poly}\left(q^{k}, m\right)$ time and find the value of $v$ which minimize this expectation. Hence, we can complete Algorithm 3 in poly $\left(q^{k}, m\right)$ time. In the following Subsection we give improvements to this algorithm, showing how to achieve the desired complexity.

\subsection{Improving the Deterministic Algorithm}

In order to find the letter $v$ which minimizes $E_{i, j, v}=E\left(\sum_{q^{j-1} \leq \ell<q^{j}} \mathcal{B}_{\delta}\left(y_{\ell}\right) \mid S T_{(i, j)}, \mathcal{G}[i, j]=v\right)$, we do not actually have to calculate the $q$ expectations $E_{i, j, v}$. It is enough to calculate the differences of those expectations and a constant value. We will use the constant value which is the expected number of bad events given $S T_{(i, j)}$ and that $\left(\mathcal{G} y_{\ell}\right)[i] \neq 0$ for all $q^{j-1} \leq \ell<q^{j}$ (Of course, it's improbable that no letters would vanish in step $(i, j)$, as the purpose of this assumption is only to help us with the proof). We denote this constant value $E_{i, j}$.

According to Lemma 5.4, for any vector $y$ the following holds:

$$
\begin{gathered}
\operatorname{Pr}\left(\mathcal{B}_{\delta}(\mathcal{G} y) \mid S T_{i, j},(\mathcal{G} y)[i]=0\right)-\operatorname{Pr}\left(\mathcal{B}_{\delta}(\mathcal{G} y) \mid S T_{i, j},(\mathcal{G} y)[i] \neq 0\right)= \\
\left(\begin{array}{c}
m-j \\
\delta m-c
\end{array}\right)\left(1-\frac{1}{q}\right)^{\delta m-c}\left(\frac{1}{q}\right)^{(m-j)-(\delta m-c)}
\end{gathered}
$$


Denote the above expression $\operatorname{Dif}_{i, j}(y)$. Let $T$ be the time it takes to calculate this expression. Now, we can calculate all $q$ differences $E_{i, j, v}-E_{i, j}$ quite efficiently in the following manner: Initialize a size $q$ array $W$. Then, run over the vectors $y_{\ell}$ for $q^{j-1} \leq \ell<q^{j}$, and for each subtract the difference $\operatorname{Dif}_{i, j}\left(y_{\ell}\right)$ from cell $v=-y_{\ell}[j]^{-1} \sum_{t=0}^{j-1} \mathcal{G}[i, t] y_{\ell}[t]$ in $W$ (since this cell means setting $\left.\left(\mathcal{G} y_{\ell}\right)[i]=\sum_{t=0}^{j-1} \mathcal{G}[i, t] y_{\ell}[t]+\mathcal{G}[i, j] y_{\ell}[j]=0\right)$. After considering all values of $y_{\ell}$, the position with the maximal value in $W$ is the letter we should set for $\mathcal{G}[i, j]$. Each entry number $v$ can be calculated in constant time for all $q^{j-1} \leq \ell<q^{j}$ if we traverse over the $\ell$-s in each step according to Gray code. Overall, the program will calculate $m q^{k}$ entries, and so, it will take $m q^{k} T$ time.

Finally, we will show how to drop the $T$ factor and achieve a $\Theta\left(m q^{k}\right)$ running time. In order to do so we need to take two measures:

- Use standard approximation techniques throughout the algorithm to approximate the weights in $W$ instead of calculating them exactly.

- Evaluate approximately all the values of $\left(\begin{array}{l}a \\ b\end{array}\right)$ for any $a \in[m], b \in[a]$ in preprocess, so that we will not need to calculate them again during the process.

After doing both changes, $T$ will drop to $\Theta(1)$.

We conclude the discussion with the following Theorem:

Theorem 2 Let $m, k, q$ be positive integers and $\delta \in[0,1]$. If $k \leq\left(1-H_{q}(\delta)\right) m$, then it's possible to construct an $[m, k, \delta m]_{q}-L C$ in time $\Theta\left(m q^{k}\right)$.

\section{Conclusion and Open Problems}

We have presented a simple and intuitive construction of linear codes meeting the GV bound. Our construction is the most efficient known construction of such linear codes. We used our codes construction to construct explicitly, in $\Theta(r n \log n)$ time, very good GT schemes of $\Theta\left(r^{2} \log n\right)$ tests. It would be interesting to study whether our linear codes construction can be made more efficient, or whether it can be improved to construct better codes. While we managed to close the gap between the sizes of explicit and non-explicit group testing schemes, the gap in the important generalization of selectors is still open; closing it is an interesting and important problem. We believe that other important special cases of group testing worth studying include the problem of minimizing the sets accumulative size rather then their number, and also, solely for algorithmic purposes - the case where the tests answers tell not only if there exists an element in the intersection or not, but rather, how many elements are there in it.

\section{References}

[1] Macula A.J. Probabilistic nonadaptive and two-stage group testing with relatively small pools and dna library screening. Journal of Combinatorial Optimization, 2:385-397(13), 1998.

[2] N. Alon and J. Spencer. The Probabilistic Method. John Wiley and Sons Inc., 2nd edition, 2001. 
[3] Noga Alon, Dana Moshkovitz, and Shmuel Safra. Algorithmic construction of sets for restrictions. ACM Transactions on Algorithms, 2(2):153-177, 2006.

[4] Amihood Amir, Oren Kapah, and Ely Porat. Deterministic length reduction: Fast convolution in sparse data and applications. In CPM, pages 183-194, 2007.

[5] D. J. Balding, W. J. Bruno, E. Knill, and D. C. Torney. A comparative survey of non-adaptive pooling designs. Institute for Mathematics and Its Applications, 81:133-+, 1996.

[6] E. Barillot, B. Lacroix, and D. Cohen. Theoretical analysis of library screening using an ndimensional pooling strategy. Nucleic Acids Research, pages 6241-6247, 1991.

[7] Toby Berger and Vladimir I. Levenshtein. Asymptotic efficiency of two-stage disjunctive testing. IEEE Transactions on Information Theory, 48(7):1741-1749, 2002.

[8] Toby Berger and Vladimir I. Levenshtein. Application of cover-free codes and combinatorial designs to two-stage testing. Discrete Appl. Math., 128(1):11-26, 2003.

[9] A. Blass and Y. Gurevich. Pairwise testing. Bulletin of the EATCS, 78:100-132, 2002.

[10] A. De Bonis, L. Gasieniec, and U. Vaccaro. Generalized framework for selectors with applications in optimal group testing. In ICALP, pages 81-96, 2003.

[11] Annalisa De Bonis, Leszek Gasieniec, and Ugo Vaccaro. Optimal two-stage algorithms for group testing problems. SIAM J. Comput., 34(5):1253-1270, 2005.

[12] Annalisa De Bonis and Ugo Vaccaro. Improved algorithms for group testing with inhibitors. Inf. Process. Lett., 67(2):57-64, 1998.

[13] Annalisa De Bonis and Ugo Vaccaro. Constructions of generalized superimposed codes with applications to group testing and conflict resolution in multiple access channels. Theor. Comput. Sci., 306(1-3):223-243, 2003.

[14] Richard A. Brualdi and Vera Pless. Greedy codes. J. Comb. Theory, Ser. A, 64(1):10-30, 1993.

[15] W.J. Bruno, D.J. Balding, E. Knill, D. Bruce, C. Whittaker, N. Dogget, R. Stalling, and D.C. Torney. Design of efficient pooling experiments. Genomics, 26:21-30, 1995.

[16] The Center for Discrete Mathematics and Theoretical Computer Science (DIMACS). DIMACS Workshop on Combinatorial Group Testing, May 2006.

[17] Shiva Chaudhuri and Jaikumar Radhakrishnan. Deterministic restrictions in circuit complexity. In ACM Symposium on Theory of Computing (STOC), pages 30-36, 1996.

[18] Mahdi Cheraghchi, Amin Shokrollahi, and Avi Wigderson. Computational Hardness and Explicit Constructions of Error Correcting Codes. In 44th Allerton Conference on Communication, Control and Computing, 2006.

[19] Marek Chrobak, Leszek Gasieniec, and Wojciech Rytter. Fast broadcasting and gossiping in radio networks. In IEEE Symposium on Foundations of Computer Science, pages 575-581, 2000 . 
[20] A.E.F. Clementi, A. Monti, and R. Silvestri. Distributed broadcast in radio networds of unknown topology. Theoretical Computer Science, 302(1-3):337-364, 2003.

[21] Andrea E. F. Clementi, Angelo Monti, and Riccardo Silvestri. Selective families, superimposed codes, and broadcasting on unknown radio networks. In SODA, pages 709-718, 2001.

[22] Raphaël Clifford, Klim Efremenko, Ely Porat, and Amir Rothschild. $k$-mismatch with don't cares. In ESA, pages 151-162, 2007.

[23] D.M. Cohen, S.R. Dalal, M.L. Fredman, and G.C. Patton. The AETG system: An approach to testing based on combinatiorial design. Software Engineering, 23(7):437-444, 1997.

[24] Graham Cormode and S. Muthukrishnan. What's hot and what's not: tracking most frequent items dynamically. ACM Trans. Database Syst., 30(1):249-278, 2005.

[25] Peter Damaschke. Randomized group testing for mutually obscuring defectives. Inf. Process. Lett., 67(3):131-135, 1998.

[26] R. Dorfman. The detection of defective members of large populations. The Annals of Mathematical Statistics, 14(4):436-440, 1943.

[27] D.Z. Du and F.K. Hwang. Combinatorial Group Testing and its Applications, volume 12 of Series on Applied Mathematics. World Scientific, 2nd edition, 2000.

[28] M. Farach, S. Kannan, E. Knill, and S. Muthukrishnan. Group testing problems with sequences in experimental molecular biology. In the Compression and Complexity of Sequences 1997, page 357, 1997.

[29] M. Farach, S. Kannan, E. Knill, and S. Muthukrishnan. Group testing problems with sequences in experimental molecular biology. In SEQUENCES '97: Proceedings of the Compression and Complexity of Sequences 1997, page 357, Washington, DC, USA, 1997. IEEE Computer Society.

[30] E.N. Gilbert. A comparison of signalling alphabets. Bell System Technical Journal, 31:504-522, 1952.

[31] V.D. Goppa. Codes associated with divisors. Problems of Information Transmission, 13(1):2226, 1977.

[32] Wassily Hoeffding. Probability inequalities for sums of bounded random variables. Journal of the American Statistical Association, 58(301):13-30, Mar. 1963.

[33] E.S. Hong and R.E. Ladner. Group testing for image compression. In Data Compression Conference, pages 3-12, 2000.

[34] W.H. Kautz and R.C. Singleton. Nonrandom binary superimposed codes. IEEE Transaction of Information Theory, 10:363-377, 1964.

[35] Knill. Lower bounds for identifying subset members with subset queries. In SODA: ACMSIAM Symposium on Discrete Algorithms (A Conference on Theoretical and Experimental Analysis of Discrete Algorithms), 1995. 
[36] Emanuel Knill, William J. Bruno, and David C. Torney. Non-adaptive group testing in the presence of errors. Discrete Applied Mathematics, 88(1-3):261-290, 1998.

[37] C.H. Li. A sequential method for screening experimental variables. J. Amer. Sta. Assoc., $57: 455-477,1962$.

[38] James L. Massey. Threshold decoding. MIT Press, Cambridge, Massachusetts, USA, 1963.

[39] H.Q. Ngo and D.Z. Du. A survey on combinatorial group testing algorithms with applications to DNA library screening. In DIMACS Series Discrete Math. and Theor. Computer Science 55, AMS 2000, pages 171-182, 2000.

[40] Pavel A. Pevzner and Robert J. Lipshutz. Towards dna sequencing chips. In MFCS '94: Proceedings of the 19th International Symposium on Mathematical Foundations of Computer Science 1994, pages 143-158, London, UK, 1994. Springer-Verlag.

[41] Benny Porat, Ely Porat, and Amir Rothschild. pattern matching in a streaming model.

[42] K. Shum. A Low-Complexity Construction of Algebric Geometry Codes Better Then the Gilbert-Varshamov Bound. PhD thesis, University of Southern California, December 2000.

[43] K. Shum, I. Aleshnikov, P.V. Kumar, H. Stichtenoth, and V. Deolalikar. A low-complexity algorithm for the construction of algebraic geometric codes better then the Gilbert-Varshamov bound. IEEE Transaction on Information Theory, 47(6):2225-2241, September 2001.

[44] M. Sobel and P.A. Groll. Group testing to eliminate efficiently all defectives in a binomial sample. Bell Syst. Tech. J., 38:1179-1252, 1959.

[45] Berger T., Mandell J.W., and Subrahmanya P. Maximally efficient two-stage screening. Biometrics, 56:833-840(8), September 2000.

[46] M.A. Tsfasman, S.G. Vladut, and T. Zink. Modular curves, Shimura curves, and codes better then the Varshamov-Gilbert bound. Math. Nachrichten, 109:21-28, 1982.

[47] R.R. Varshamov. Estimateof the number of signals in error correcting codes. Doklady Akadamii Nauk, 117:739-741, 1957.

[48] J.K. Wolf. Born again group testing: Multiaccess communications. IEEE Transactions on Information Theory, 31(2):185-191, 1985.

[49] J.M. Wozencraft. Threshold decoding. Personal communication in [38] section 2.5, 1963. 